\title{
CONSTRICTIVE PERICARDITIS: AN OVERLOOKED CAUSE OF ASCITES
}

\author{
Beliz Koçyiğit ${ }^{1}$ (D), Irmak İrem Özyiğit ${ }^{1}$ (D), Servet Altay $^{2}$ (D)
}

${ }^{1}$ Trakya University School of Medicine, Edirne, TURKEY

${ }^{2}$ Department of Cardiology, Trakya University School of Medicine, Edirne, TURKEY

\begin{abstract}
Aims: Constrictive pericarditis is a well-known but unusual and rare cause of ascites. The diagnosis of constrictive pericarditis in most patients who have chronic ascites is challenging. We aimed to present a patient with constrictive pericarditis with chronic ascites and pericardial calcifications seen in chest radiography. Case Report: A 66-year-old male patient presented to the emergency room of Trakya University School of Medicine. The patient had abdominal swelling and ascites for the last 5 years and underwent large-volume paracentesis in his previous emergency admissions. After the electrocardiography of the patient, he was directed to the cardiology department for further examinations. The chest radiography revealed significant pericardial calcifications. Following imaging procedures confirmed radiological findings, and the patient was diagnosed with constrictive pericarditis. Furthermore, his cardiac catheterization findings were consistent with constrictive pericarditis. The patient was recommended to have a pericardiectomy operation and there was a significant reduction in the patient's symptoms after surgery. Conclusion: The absence of specific symptoms and resemblances of existing symptoms to liver disease make the early diagnosis of constrictive pericarditis difficult. Physicians should consider constrictive pericarditis as a differential diagnosis when dealing with a patient who has chronic ascites. Keywords: Constrictive pericarditis, ascites, pericardial disease
\end{abstract}

\section{INTRODUCTION}

Constrictive pericarditis $(\mathrm{CP})$ results from pericardial fibrous thickening and reveals itself with restrained diastolic function caused by inelastic pericardium $(1,2)$. Throughout history, the main cause of CP remains to be tuberculosis and is still highly seen in particular countries, mostly ones that are not fully industrialized and with immunodeficiency-related population profiles (1, 2 ). In developed countries, the unknown antecedent is the leading cause of CP (1). The prevalent cause of CP in Turkey is tuberculosis, which supports the literature (2).

Ascites, edema, and hepatomegaly are the common symptoms of CP (3). A combination of hepatomegaly and ascites can be observed through the physical examination of the abdomen (2). When ascites and hepatomegaly are present together in a patient, misdiagnosis of a liver-related disease is common (1). Due to the challenges in differentiating by these primary signs, diagnosis of CP can remain unnoticed by physicians (4). Lack of typical cardiological signs causes referrals to non-cardiological specialties, which delay diagnosis and treatment $(4,5)$.

Though it is difficult to differentiate; anamnesis, biochemical parameters, unexplained examination findings, and imaging techniques can reveal CP when considered altogether (2). Chest radiographs, computed tomography scanning, cardiac magnetic resonance imaging (MRI), cardiac catheterization, and echocardiograms can lead physicians to a final diagnosis if a patient has findings consistent with CP (1-3).

Treatment of CP includes different approaches based on the type of disease such as chronic or pericardial inflammation ap- proaches (2). Anti-inflammatory therapy is needed when patients are present with inflammatory clinical findings, while chronic $\mathrm{CP}$ requires pericardiectomy surgery by its progressive nature $(1,2)$. Patients' symptoms are significantly relieved in the postoperative course (2).

We aim to present a case report regarding a patient with $\mathrm{CP}$ with a history of chronic ascites who underwent a pericardiectomy operation. Since the differential diagnosis of CP as a cause of ascites can be challenging, we believe this case will contribute to the existing literature.

\section{CASE REPORT}

A 66-year-old male patient presented to the emergency department of Trakya University School of Medicine with the complaint of abdominal swelling. It was known that the patient applied to the emergency service with the complaint of abdominal swelling and ascites for about 5 years. At his emergency admissions, he underwent large-volume paracentesis to remove ascites. The patient was followed up by the gastroenterology department with a diagnosis of cardiac cirrhosis. The patient was a current smoker (30 packs/year) with a history of hypertension, hepatitis B, umbilical hernia operation, and appendectomy. The patient was also using $110 \mathrm{mg}$ twice daily dosing of dabigatran, $5 \mathrm{mg}$ of ramipril, $25 \mathrm{mg}$ of spironolactone, and $40 \mathrm{mg}$ of furosemide. According to the patient's hemogram, his erythrocyte count was 3900000/uL, his hemoglobin was $11.6 \mathrm{~g} / \mathrm{dL}$, and his hematocrit was $35.2 \%$. The patient had neutrophilia $(5700 / \mathrm{uL})$ and lymphocytopenia $(800 / \mathrm{uL})$. The patient's C-reactive protein 
level was elevated $(1.34 \mathrm{mg} / \mathrm{dL})$, his total protein $(6.5 \mathrm{~g} / \mathrm{dL})$, and albumin $(3.4 \mathrm{~g} / \mathrm{dL})$ levels were slightly low. The patient's transaminase levels were within the normal range.

Atrial fibrillation was detected in the electrocardiography and the patient was directed to the cardiology department with the pre-diagnosis of heart failure. On physical examination, palpitations and ascites were detected in the patient, and he was pre-diag-

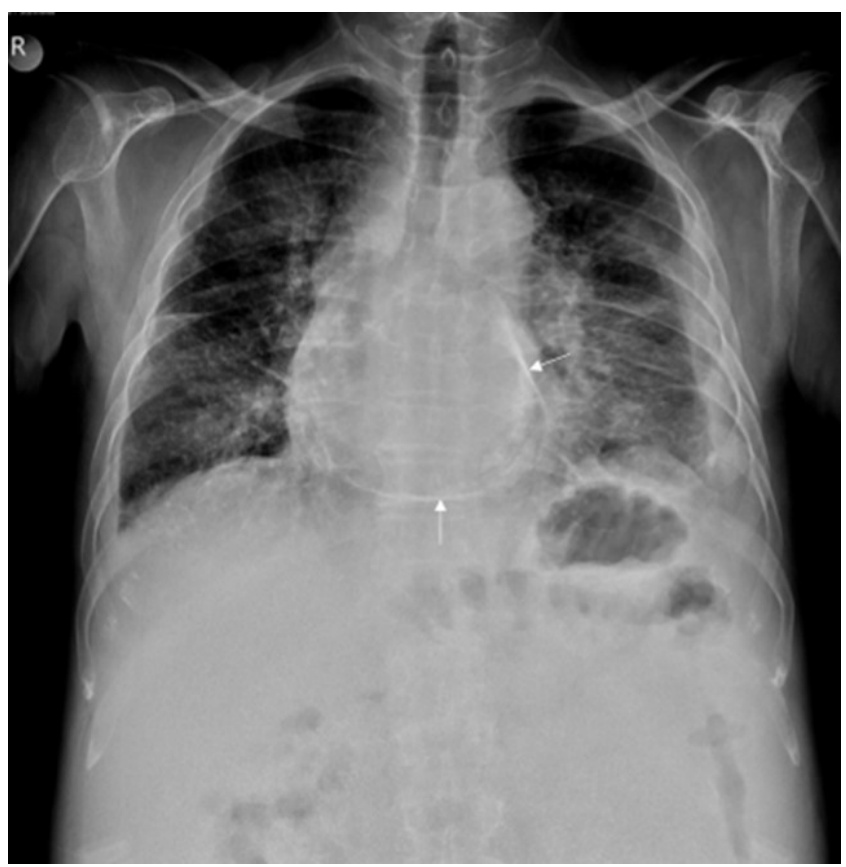

Figure 1: Posteroanterior chest radiography revealing pericardial calcifications (Arrows).


Figure 2: A, B: Cardiac MRI (short axis T1A view) of the heart shows pericardial thickening more pronounced over the left ventricle. C: A closer image of A. Thickness measured as $5.2 \mathrm{~mm}$ (Line). D: A closer image of B (Line). nosed with heart failure. Significant pericardial calcifications were observed in the posteroanterior chest radiography of the patient (Figure 1). In the echocardiography, pulmonary pressure was 55 $\mathrm{mm} \mathrm{Hg}$ and the thickness of the inferior vena cava was $26 \mathrm{~mm}$. Mild tricuspid regurgitation, mild mitral insufficiency, biatrial enlargement, and pulmonary hypertension were detected in the patient. Later on, the patient was directed to cardiac MRI with suspicion of CP. Cardiac MRI showed localized pleural effusions on both sides, prominent on the right, and intra-abdominal diffuse intraperitoneal fluid. Right heart chambers were larger than normal, and the vena cava was wider than normal, $43 \mathrm{~mm}$ at the inferior suprahepatic level. There was biatrial dilatation in the heart and the pericardium was measured as $5.2 \mathrm{~mm}$ in its most prominent place adjacent to the left ventricle. The thickening of the pericardium is shown in Figure 2. Cardiac MRI results also strengthened the pre-diagnosis of CP. All of these imaging findings supported the diagnosis of CP. Our patient was then referred to the coronary angiography laboratory for right left heart catheterization with the indication of CP. Cardiac catheterization revealed equalization of the end-diastolic pressure of the right and left ventricles, square root sign, and enlargement of the cardiac silhouette. Calcification and thickening were also observed in the pericardium. It was reported that the patient had catheter and fluoroscopy findings consistent with CP.

Subsequently, our patient was diagnosed with constrictive pericarditis and he was transferred to the cardiovascular surgery department for a pericardiectomy operation. During surgery, it was observed that the pericardium was adhered to the pleura, heart, and surrounding tissues and was highly calcified. There was a significant reduction in the patient's symptoms after surgery.

\section{DISCUSSION}

Historically, tuberculosis has been cited as the most common cause of CP (2). On a global scale, this is still the case, especially in regions where the human immunodeficiency virus and acquired immunodeficiency syndrome are very common (2). While tuberculosis is still the leading cause of CP in developing countries, it is now relatively rare in the United States and Europe $(2,6)$. The leading etiologies of CP in developed countries are idiopathic, post-cardiac surgery, and radiation $(2,6,7)$. In our case, the patient did not have any of these prior conditions, however, he had a history of hepatitis B. Even though it is not as common as the previously mentioned etiologies, hepatitis is one of the pathogens that can lead to $\mathrm{CP}$ (8). It is also possible that even though it was not known, he could have had a history of undiagnosed tuberculosis since it is still the leading cause in developing countries.

Constrictive pericarditis may present with signs and symptoms of left heart failure such as dyspnea on exertion, orthopnea, bilateral pleural effusion, pulmonary edema, and with signs and symptoms of right heart failure such as hepatomegaly, ascites, peripheral edema, and hepatic congestion $(2,9)$. Due to varying clinical manifestations and initial history, patients may be evaluated for liver disease before being referred to cardiological evaluation. Our patient also had an ongoing history of abdominal swelling and ascites and was previously diagnosed with cardiac cirrhosis.

Although the electrocardiogram might be unremarkable when it comes to diagnosis, atrial fibrillation was reported in approximately $20-40 \%$ of the patients, which was also present in our patient (10). Pericardial calcifications may be a very helpful finding in chest radiographs, especially in idiopathic cases (2). In a case series with a large number of patients, pericardial calcifications were prevalent in $27 \%$ of the patients (11). This was also a crucial finding 
in our case since it led us to consider $\mathrm{CP}$ as the cause of ascites. The Mayo Clinic echocardiography diagnosis study found that the three prominent variables associated with $\mathrm{CP}$ were (i) ventricular septal shift, (ii) medial mitral e' (early diastolic mitral annulus velocity), and (iii) hepatic vein expiratory diastolic reversal ratio (12). In our case, the echocardiography showed lateral e' as $7 \mathrm{~cm} / \mathrm{s}$ and septal e' as $14 \mathrm{~cm} / \mathrm{s}$. It also showed $26 \%$ of inferior vena cava collapse which indicated inferior vena cava plethora. Inspiratory reverse flow increase and septal bounce were positive. Although our patient's echocardiography results did not completely meet the Mayo Clinic criteria, some of the findings such as the medial e' velocity and inferior vena cava plethora led us to consider possible CP (10). Some of the characteristic findings in the MRI are pericardial thickening, pericardial calcifications, and pericardial effusions (10). Pericardial thickening and pleural effusions were also present in our patient, which was found to be in line with the literature. Hemodynamic catheterization, which remains the gold standard diagnostic tool for CP, may be used when non-invasive tests are inconclusive or indeterminate $(2,10,13)$. Expected findings on cardiac catheterization such as equalization of the end-diastolic pressure of the right and left ventricles and square root sign were present in our patient, and therefore led us to a conclusive diagnosis $(10,13)$.

Our patient suffered from ascites for about 5 years, which was thought to be caused by liver disease. CP is a well-known but unusual and rare cause of ascites $(5,11)$. Due to the lack of specific symptoms and liver disease resemblances, the early diagnosis of CP can be challenging (5). Physicians should consider CP as a differential diagnosis when dealing with a patient who has chronic ascites. We hope that this case will help raise awareness among physicians and shine more light on the condition.

Ethics Committee Approval: N/A

Informed Consent: Informed verbal consent was obtained from the patient. Conflict of Interest: The authors declare no conflict of interest.

Author Contributions: Concept: BK, IIIÖ, SA. Design: BK, IİO, SA. Supervision: BK, IIÖ, SA. Resources: BK, IİO, SA. Materials: BK, IİÖ, SA. Data collection and/or Processing: BK, IİÖ, SA. Analysis and/or Interpretation: BK, IİO, SA. Literature Search: BK, IİÖ, SA. Writing Manuscript: BK, IİÖ, SA. Critical Review: BK, IIIÖ, SA.
Financial Disclosure: The authors declared that this study received no financial support.

Acknowledgments: We would like to thank Fethi Emre Ustabaşığlu from the Radiology Department of Trakya University School of Medicine for their valuable guidance and contributions.

Editor-in-Chief's Note: Two of the authors of this article, Beliz Koçyiğit and Irmak İrem Özyiğit are members of the editorial board of Turkish Medical Student Journal. However, they did not take place at any stage of the editorial decision of the manuscript. The editors who evaluated this manuscript are from other institutions.

\section{REFERENCES}

1. Fowler NO. Constrictive pericarditis: its history and current status. Clin Cardiol 1995; 18(6):341-50

2. Welch TD, Oh JK. Constrictive pericarditis. Cardiol Clin 2017;35(4):539-49.

3. Sengupta PP, Eleid MF, Khandheria BK. Constrictive pericarditis. Circ J 2008;72(10):1555-62.

4. Barry M, Al-Muhaidb S, Fathala A. A case report of constrictive pericarditis: a forgotten cause of refractory ascites. Radiol Case Rep 2020;15(12):2565-8.

5. Howard JP, Jones D, Mills P et al. Recurrent ascites due to constrictive pericarditis. Frontline Gastroenterol 2012;3(4):233-7.

6. George TJ, Arnaoutakis GJ, Beaty CA et al. Contemporary etiologies, risk factors, and outcomes after pericardiectomy. Ann Thorac Surg 2012;94(2):445-51.

7. Miranda WR, Oh JK. Constrictive pericarditis: a practical clinical approach. Prog Cardiovasc Dis 2017;59(4):369-79.

8. Griffin BP, Topol EJ. Pericardial disease. Manual of Cardiovascular Medicine. 2nd ed. Philadelphia, Pennsylvania: Lippincott Williams and Wilkins; 2004.p.372-96.

9. Chang SA, Oh JK. Constrictive pericarditis: a medical or surgical disease? J Cardiovasc Imaging 2019;27(3):178-86.

10. Welch TD. Constrictive pericarditis: diagnosis, management and clinical outcomes. Heart 2017;104(9):725-31.

11. Ling LH, Oh JK, Breen JF et al. Calcific constrictive pericarditis: is it still with us? Ann Intern Med 2000;132(6):444-50.

12. Welch TD, Ling LH, Espinosa RE et al. Echocardiographic diagnosis of constrictive pericarditis: Mayo Clinic criteria. Circ Cardiovasc Imaging 2014;7(3);526-34.

13. Syed FF, Schaff HV, Oh JK. Constrictive pericarditis-a curable diastolic heart failure. Nat Rev Cardiol 2014;11(9):530-44. 